\title{
Disagreement between parents and health professionals regarding pain intensity in critically ill neonates
}

\author{
Luciana S. D. T. Elias, ${ }^{1}$ Ruth Guinsburg, $^{2}$ Clóvis A. Peres, $^{3}$ Rita C. X. Balda, ${ }^{4}$ \\ Amelia M. N. dos Santos 5
}

\begin{abstract}
Objective: To verify whether parents and health professionals homogeneously evaluate presence and intensity of neonatal pain.

Methods: This cross-sectional study enrolled 52 neonates and 154 adults. Inclusion criteria for neonates were admission to neonatal intensive care unit, presence of gastric tube, tracheal tube, and venous lines. Each newborn was observed by a different group of three adults (parent, nurse assistant and pediatrician) for 1 minute at the same time to evaluate presence and intensity of infant's pain. Homogeneity of pain evaluation was analyzed by a modified BlandAltman plot and by intraclass correlation coefficient (ICC). Multiple linear regression analysis was used to evaluate association of neonatal characteristics and heterogeneity of pain scores for adults.

Results: ICC showed disagreement of the pain scores given by the three groups of adults (ICC 0.066 , agreement $>0.75$ ). Bland-Altman analysis showed agreement among adults when they thought pain was absent. When they thought pain was present, there was heterogeneity of opinions regarding intensity of neonatal pain. Multiple regression analysis indicated that $10 \%$ of this disagreement could be explained by infant's gender and mode of delivery.
\end{abstract}

Conclusions: Disagreement among adults about intensity of neonatal pain is a marker of the difficulty in deciding the need for analgesia in preverbal patients.

J Pediatr (Rio J). 2008;84(1):35-40: Pain, pain measurement, infant, newborn.

\section{Introduction}

Adults should recognize and interpret the signs shown by neonates after a painful or stressful stimulus, since it is through such signs as facial expression, body movement, crying, and state of consciousness, among others, that infants establish an interpersonal communication process, which is their pain "language."

Such coding and decoding mechanism used by health professionals and parents, who subsequently make the decision on how pain should be handled, is not a simple process. It may be influenced by a number of factors related to the characteristics of observers themselves, such as age, sex, race, religion, marital status, socioeconomic level, previous personal or family experience with pain, professional background and experience. ${ }^{1-9}$ In addition to the characteristics of the pain observer, factors inherent to the patient, such as gestational age, sex, race, physical appearance, presence of tissue damage, and severity of clinical-surgical diagnosis, may also

1. Médica. Doutoranda em Pediatria, Divisão de Pediatria Neonatal, Universidade Federal de São Paulo - Escola Paulista de Medicina (UNIFESP-EPM), São Paulo, SP, Brazil.

2. Professora titular, Divisão de Pediatria Neonatal, UNIFESP-EPM, São Paulo, SP, Brazil.

3. Professor titular, Departamento de Epidemiologia, UNIFESP-EPM, São Paulo, SP, Brazil.

4. Doutora. Médica, Divisão de Pediatria Neonatal, UNIFESP-EPM, São Paulo, SP, Brazil.

5. Doutora. Professora associada, Divisão de Pediatria Neonatal, UNIFESP-EPM, São Paulo, SP, Brazil.

Disciplina de Pediatria Neonatal do Departamento de Pediatria da Universidade Federal de São Paulo, Escola Paulista de Medicina.

No conflicts of interest declared concerning the publication of this article.

Suggested citation: Elias LS, Guinsburg R, Peres CA, Balda RC, dos Santos AM. Disagreement between parents and health professionals regarding pain intensity in critically ill neonates. J Pediatr (Rio J). 2008;84(1):35-40.

Manuscript received Sep 28 2007, accepted for publication Nov 082007.

doi:10.2223/JPED.1748 
change the inference of presence and magnitude of pain by the observer. ${ }^{1,3,5-8,10}$

Studies that investigate these complex interactions may help simplify the proper approach to the diagnosis and treatment of pain in preverbal patients. Therefore, this investigation aimed at verifying whether parents and health professionals homogeneously or heterogeneously evaluate presence and magnitude of pain in critically ill newborn infants.

\section{Methods}

This cross-sectional study was carried out in the neonatal intensive care units (NICU) of three hospitals coordinated by Universidade Federal de São Paulo (UNIFESP), located in São Paulo, Brazil, from July 2001 through September 2002, after approval by the institutional Independent Ethics Committee.

After obtaining written informed consent, neonates were enrolled if they met the following inclusion criteria: any gestational age and birth weight, with 24 to 96 hours of life, and under the following care: in an incubator, with a gastric tube, a peripheral and/or a central venous access and in conventional mechanical ventilation by endotracheal tube, regardless of ventilation parameters and use of vasopressors. Patients could not have congenital anomalies.

Adults to be interviewed were selected according to the following groups:

Group 1: father or mother of all infants that met the inclusion criteria, provided that they were not health professionals. Adults in this group were selected for interview during parents' visit to the newborn infant (convenience sample).

Group 2: technical nurses that were working in one of the three NICU. Each nurse evaluated a single newborn included in the study, which could not be under her direct care at that shift. Adults in this group were randomly selected (sealed envelopes) among all nursing staff not assigned to that patient's care and willing to participate in the study. If the technical nurse had already evaluated one study patient, she was excluded from the selection.

Group 3: pediatricians, provided that they did give direct assistance to the newborn included in the study. Each pediatrician evaluated a single newborn. Adults in this group were randomly selected (sealed envelopes) among the medical staff not assigned to that patient's care and willing to participate in the study. Again, physicians that had already participated in the research were excluded from the selection.

Sample size was calculated to verify which factors related to the newborns were associated with agreement or disagreement regarding presence and/or intensity of pain in these neonates among the adults groups. Considering the need of 10-15 individuals for each variable entered in multiple regression models, ${ }^{11} 10$ three-adult groups (each three-adult group was formed by one parent, one nurse and one pediatrician) were required for each factor related to the newborn possibly associated with agreement/disagreement on neonatal pain evaluation. There were initially five characteristics of interest: mode of delivery, gender, birthweight, gestational age, use of opioids. Therefore, it was planned that 50 three-adult groups (150 adults) observe each different baby.

Demographic and clinical information of enrolled infants was collected, and the interviews with the three adults, for each baby observed, were performed at 1-hour intervals at the most, so that adults would not observe the same baby at different moments of clinical evolution. Each adult answered a questionnaire containing the following information:

- Demographic and professional information: age, gender, race, marital status, religion, number of children, education, occupation, and monthly per capita income.

- Questions proposed to evaluate pain: the interviewee was placed in front of the neonate and was allowed 1 minute to observe it. At the completion of this period, the following question was asked: "Do you think the newborn (or your child) is in pain? For affirmative responses, the following was asked: "How much pain do you think the newborn (or your child) feels?" The answer was marked with an X in the visual analog scale held by the interviewee (a scale made of a $10-\mathrm{cm}$ vertical and not numbered line, with a trace in the lower end written "absence of pain," and, in the upper end, another trace with the indication "worst imaginable pain"). At the completion of all interviews the distance in centimeters was measured from point zero ("absence of pain") to the point marked by the observer.

The software SPSS (11.0) was used in all statistical analysis, being significant $p \leq 0.05$. Categorical variables were compared by chi-square test and its partition, and numeric variables by analysis of variance (ANOVA), with the differences located by Bonferroni method.

For the study of homogeneity or heterogeneity of the evaluation of pain among the three-adult groups interviewed for each baby, a modified Bland-Altman analysis was performed. ${ }^{12}$ The Bland-Altman plot allowed visual observation of the relation between the standard deviation and the mean score of pain attributed by each three-adult group for each baby observed. When there was agreement between observers, the standard deviation around the mean value was close to zero and, as disagreement increased, the standard deviation also increased. Therefore, when disagreement between observers was significant, there were 2 or more standard deviations around the mean neonatal pain score given by the three adults. In addition, the intraclass correlation coefficient (ICC) was applied to verify whether the groups as a whole agreed in relation to the scores of pain attributed to the neonates. ${ }^{13}$

To study the possible neonatal factors associated with agreement or disagreement of evaluation of pain among the 
adults, the relation between each variable of the newborn was individually analyzed in relation to the standard deviation of the mean score of pain attributed by the adults groups. Linear regression analysis was used for numeric variables and ANOVA for the categorical ones. A multiple linear regression model was then built and variables with a significance level higher than $25 \%$ were stepwise excluded, with the backward strategy. $R$ value was calculated to show how much of the variation of the dependent variable could be explained by the independent variables of the final regression model. ${ }^{12}$

\section{Results}

At the units, during the study period, 54 neonates met the inclusion criteria, including presence of three adult observers available for the concurrent evaluation of pain (one physician, one nurse and either parent). Only two of these 54 patients were not evaluated due to mother refusal (one patient) and physician refusal (one patient).

Regarding the 52 studied newborns, 35 (67\%) were delivered by $\mathrm{C}$-section. Mean 1- and 5-minute Apgar scores were $6(0-10)$ and $8(1-10) ; 33(64 \%)$ neonates were male. Mean birth weight, gestational age and postnatal age were $1,530 \mathrm{~g}$ (605-4,270), 32 weeks (25-42) and 42 hours (24-96) of life. The main diseases responsible for NICU admission were pulmonary problems in 34 (65\%), early onset sepsis in nine $(17 \%)$, and hypoxic-ischemic encephalopathy in three (6\%). At the time of pain evaluation, all patients had central and/or peripheral venous access, and were mechanically ventilated for a mean of 33 hours (8-94). Concerning medication, 44 (85\%) newborns were receiving antibiotics, 32 (62\%) were under dopamine infusion, 23 (44\%) on dobutamine, 20 (39\%) on both, eight (15\%) on midazolam infusion, and 47 (90\%) were on opioids at the time of evaluation.
The 52 newborns were evaluated by the three-adult groups: group 1 comprised by two fathers and 48 mothers, group 2 by 52 technical nurses, and group 3 by 52 pediatricians. The parents were interviewed during their visits to the NICU. No health professional was interviewed more than once. In case of twins, which occurred twice (four newborns), the mothers evaluated each baby at different times.

General characteristics of the adults interviewed for the three groups are shown in Table 1 . Out of the 154 adults interviewed, $16(32 \%)$ parents, $20(38 \%)$ nurses and 19 (37\%) physicians thought the newborn was in pain at the time of evaluation. There was no statistical difference between the three groups of adults $\left(x^{2} ; p=0.694\right)$. Mean score of pain attributed by the group of parents was $1.2 \pm 2.1$ (median 0 ; variation $0-8.7$ ), $1.5 \pm 2.4$ for nurses (median 0 , variation $0-10$ ), and $1.4 \pm 2.2$ for physicians (median 0 ; variation $0-7$ ), without statistical difference between groups (ANOVA, $\mathrm{p}=$ 0.837) (Table 1).

To verify the heterogeneity or homogeneity of the evaluation of each baby by the three-adult groups, a modified Bland-Altman analysis evaluated the relation between the mean score for each three-adult group ( $x$ axis, Figure 1 ) and the standard deviation of such evaluation ( $y$ axis, Figure 1) for each of the 52 observed newborns. For "absence of pain," there was agreement between the three-adult groups: 16 points related to the 16 (31\%) babies of the 52 evaluated by the three-adult group, around zero in Figure 1. When observers thought that the patient was in pain, heterogeneity was noted in this evaluation. If the mean score of pain attributed by the observers was equal to or higher than 1.5 , the standard deviation of these mean scores, in most cases, was above 2 standard deviations, indicating a disagreement between adults. These findings are reinforced by the ICC of 0.066

Table 1 - General characteristics of the three groups of adults

\begin{tabular}{|c|c|c|c|c|}
\hline & $\begin{array}{c}\text { Parents } \\
\mathbf{n}=\mathbf{5 0}\end{array}$ & $\begin{array}{c}\text { Nurses } \\
n=52\end{array}$ & $\begin{array}{l}\text { Pediatricians } \\
\quad n=52\end{array}$ & $\mathbf{p}$ \\
\hline Age (years) & $28 \pm 8$ & $3 \pm 8$ & $31 \pm 8$ & $0.027^{*}$ \\
\hline Female, $\mathrm{n}(\%)$ & $48(96 \%)$ & $52(100 \%)$ & $44(85 \%)$ & $0.004^{\ddagger}$ \\
\hline White, n (\%) & $23(46 \%)$ & $34(65 \%)$ & $45(87 \%)$ & $<0.0001^{\ddagger}$ \\
\hline Catholic, n (\%) & $32(64 \%)$ & $21(40 \%)$ & $36(69 \%)$ & $0.007^{\ddagger}$ \\
\hline Stable partner, n (\%) & $37(74 \%)$ & $24(46 \%)$ & $16(31 \%)$ & $<0.0001^{\ddagger}$ \\
\hline Number of children & $2.2 \pm 2.0$ & $0.9 \pm 1.0$ & $0.2 \pm 1.0$ & $<0.0001 * \S$ \\
\hline$\geq 11$ years of school, $n(\%)$ & $10(19 \%)$ & $50(96 \%)$ & $52(100 \%)$ & $<0.0001^{\ddagger}$ \\
\hline Monthly per capita income (U\$) & $313 \pm 520$ & $800 \pm 370$ & $2,600 \pm 1,850$ & $<0.0001 * 11$ \\
\hline
\end{tabular}

\footnotetext{
* ANOVA.

${ }^{+}$Bonferroni test, $\mathrm{p}<0.05$ : parents $<$ nurses = pediatricians.

₹ Chi-square test.

$\S$ Bonferroni test, $\mathrm{p}<0.05$ : parents $>$ nurses $=$ pediatricians.

" Bonferroni test, $\mathrm{p}<0.05$ : parents $<$ nurses $<$ pediatricians.
} 
(CI95\% -0.0839-0.2496), far from 0.75, the number that indicates agreement between groups. ${ }^{12}$

All newborns enrolled in this study were under intensive care, as specified in the inclusion criteria. In addition to the standard intensive care, the neonates presented individual and not previously selected characteristics, such as birth weight, gestational age, postnatal age, Apgar scores, gender, delivery mode, use or not of opioids, sedatives, antibiotics, and vasopressors at the time of observation. The relationship between the heterogeneity index used above (the standard deviation of mean scores of pain attributed by the three-adult groups) and the neonatal characteristics that could differ among patients was studied. There was no relation among the continuous variables and the heterogeneity of the evaluation of pain among the three-adult groups for birth weight $(r=0.165 ; p=0.241)$, gestational age $(r=$ $0.132 ; p=0.353)$, postnatal age $(r=0.097 ; p=0.496)$, Apgar score at 1 minute $(r=0.053 ; p=0.707)$, and hours of mechanical ventilation $(r=0.001 ; p=0.994)$. Likewise, there was no relation among the categorical variables and the heterogeneity of observation of pain between the three-adult groups for delivery mode (ANOVA, $p=0.086$ ), gender (ANOVA, $p=0.156$ ), presence or not of peripheral venous access (ANOVA, $p=0.493$ ), use or not of antibiotics (ANOVA, $p=0.602$ ), vasopressors (ANOVA; $p=0.555$ ), sedatives (ANOVA, $p=0.576$ ) or opioids (ANOVA, $p=0.487$ ).

A multiple linear regression model was built, considering as dependent variable the standard deviation of mean scores of pain attributed by the adult physician, nurse, and father/ mother. As independent variables, postnatal age, Apgar score at 1 minute, delivery mode, gender, presence of peripheral venous access and infusion of opioids were tested. Final model is shown in Table 2 and has the following variables: delivery mode $(p=0.061)$, gender $(p=0.086)$ and the interaction

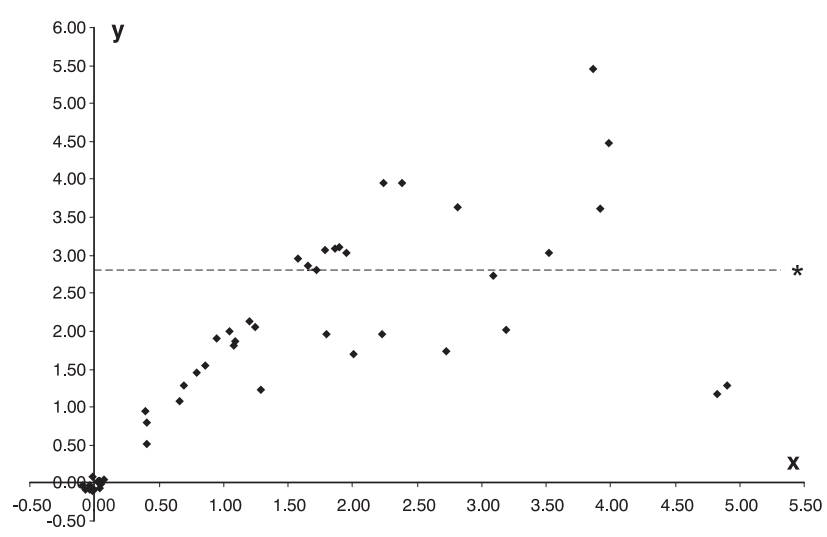

* Dotted line showing 2 standard deviations

Figure 1 - Relation between the standard deviation of mean score of pain attributed by the three-adult groups ( $y$ axis) and the average of these three pain scores ( $x$ axis) for each of the 52 studied infants between them $(p=0.052)$. According to this model, for neonates born by vaginal delivery there was more heterogeneity of pain evaluation for female infants, compared to males. Disagreement between adults was also larger for infants born via vaginal compared to those delivered by $\mathrm{C}$-section, but for those born by $\mathrm{C}$-section, there were no differences between genders.

This model explained only $10 \%(R=0.0102)$ of the difference in the evaluation of pain between the three-adult groups.

\section{Discussion}

This study is different from others available in the literature because the problem investigated was not related to the assessment of neonatal pain during a stressful or painful procedure, but to the assessment of homogeneity or heterogeneity in the adults' evaluation of neonatal pain in a standard NICU situation. In this sense, presence of pain in each baby observed was not the main object of the study, but whether, at the time of the observation, the three-adult groups agreed or disagreed on presence and magnitude of pain the newborn was possibly in.

Among the tools available to evaluate pain, the visual analog scale was chosen because it is a simple, easily applicable, understandable, effective and noninvasive method, which can be used at the bedside and help quantify the subjective evaluation of the sensation of pain each adult thought the newborn might feel at that time. The visual analog scale meets the requirements of the definition of pain of the International Association for the Study of Pain. ${ }^{14}$

There was agreement on the part of physicians, nurses, and parents concerning absence of pain in the newborns observed. On the other hand, as the average score of pain marked by the three-adult groups departed from zero, there was a growing disagreement between observers, particularly in cases of moderate pain, with scores from 1.5-5.0. Thus, when it comes to deciding whether the pain needs to be treated, the adults interfering or making this decision do not agree between themselves. Such finding matches previous works indicating that there are differences in adult evaluation of pain in other adults or children according to personal, professional, emotional or affective characteristics of observers. ${ }^{2,15-20}$

"Important" adults to make decisions regarding indication of analgesia in critically ill newborn infants disagree in relation to "how much pain" the baby is feeling. This means there is heterogeneity between adults to conclude whether the pain eventually felt by the patient is intense enough to "deserve" a therapeutic approach. The heterogeneity of magnitude of pain evaluation observed here and the implications in the communication between different health professionals and parents of critically ill babies point to the need of using validated, multidimensional pain evaluation methods for newborn infants, in which the subjective impressions of each adult 
Table 2 - Neonatal factors associated with the heterogeneity of pain evaluation of the 52 neonates by the three-adult groups

\begin{tabular}{lccc}
\hline & Degrees of freedom & F values & p \\
\hline Mode of delivery & $1 ; 204$ & 3.675 & 0.061 \\
Gender & $1 ; 204$ & 3.065 & 0.086 \\
Delivery x gender & $1 ; 204$ & 3.976 & 0.052 \\
\hline
\end{tabular}

ANOVA for the model: $p=0.043 ; R=0.0102$.

involved in the caretaking of that newborn remains in the background. ${ }^{21}$

But it is important to recognize the limits of our findings. This study does not comprise an intense pain situation, in which doubtlessly analgesia is always recommended. Once the newborns were observed during a NICU standard condition and not during an acute painful procedure, the homogeneity or heterogeneity of pain evaluation by the three-adult groups in this last situation should be the object of new studies. For the two babies in whom the average score of pain marked by adults was higher, disagreement between the three adults was lower. There seemed to have been more agreement between the observers in extreme situations: absent pain, and perhaps intense pain. In intermediary situations heterogeneity was noted in the evaluation of pain of the critically ill newborn on the part of parents, nurses, and physicians.

Several studies in the literature indicate that patientrelated factors could interfere with the evaluation of pain made by the observer. ${ }^{3,5-8,10,16-18}$ In this investigation, we speculated whether the characteristics inherent to the babies could be associated with the divergence of opinion of the threeadult group in relation to the pain possibly felt by the same infant. The multiple regression analysis showed that the variables gender, delivery mode and interaction between both were significantly associated with the heterogeneity of pain evaluation by the three-adult groups in neonates under intensive care. In view of a female patient born by vaginal delivery, the divergence of the evaluation of pain was higher between the three-adult groups than for a male baby or for those born by $\mathrm{C}$-section. There seems to be a different look and/or care on the part of adults according to the child gender, ${ }^{5,6,8}$ and perhaps the plastic phenomena more evident in the vaginal delivery changed the visual impression the adult had of each newborn, ${ }^{22,23}$ interfering with the evaluation of pain. Anyway, only $10 \%$ of the disagreement between adults could be explained by factors that are inherent to the babies.

In view of the multiple factors that are hard to control and may influence heterogeneity of the evaluation of pain possibly felt by newborn infants, use of validated, relatively objective and uniform pain evaluation tools is recommended for the entire team engaged in the NICU.

\section{References}

1. Balda RC, Guinsburg R. Perceptions of neonatal pain. Neoreviews. 2007; 8: e533-42.

2. Xavier Balda R, Guinsburg R, de Almeida MF, Peres C, Miyoshi MH, Kopelman BI. The recognition of facial expression of pain in full-term newborns by parents and health professionals. Arch Pediatr Adolesc Med. 2000;154:1009-16.

3. Breau LM, McGrath PJ, Stevens B, Beyene J, Camfield CS, Finley GA, et al. Judgments of pain in the neonatal intensive care setting: a survey of direct care staffs' perceptions of pain in infants at risk for neurological impairment. Clin J Pain. 2006; 22:122-9.

4. Hall CW, Gaul L, Kent M. College students' perception of facial expressions. Percept Mot Skills. 1999;89(3 Pt 1):763-70.

5. He HG, Pölkki T, Pietilä AM, Vehviläinen-Julkunen K. Chinese parent's use of nonpharmacological methods in children's postoperative pain relief. Scand J Caring Sci. 2006;20:2-9.

6. Hobara M. Beliefs about appropriate pain behavior: cross cultural and sex differences between Japanese and Euro-Americans. Eur J Pain. 2005;9:389-93.

7. Pillai Riddell RR, Craig KD. Judgments of infant pain: impact of caregiver identity and infant age. J Pediatr Psychol. 2007; 32:501-11.

8. Robinson ME, Wise EA. Prior pain experience: influence on the observation of experimental pain in men and women. J Pain. 2004;5:264-9.

9. Wilson B, McSherry W. A study of nurses' inferences of patients' physical pain. J Clin Nurs. 2006;15:459-68.

10. Green C, Todd KH, Lebovits A, Francis M; American Academy of Pain Medicine Council on Ethics. Disparities in pain: ethical issues. Pain Med. 2006;7:530-3.

11. Kleinbaum DG, Kupper LL. Applied regression analysis and other multivariable methods. Boston: PWS-Kent; 1988.

12. Bland JM, Altman DG. Statistical methods for assessing agreement between two methods of clinical measurement. Lancet. 1986;1:307-10.

13. Conover WJ. Practical nonparametric statistics. New York: Wiley; 1999.

14. Pain terms: a list with definitions and notes on usage. Recommended by the IASP Subcommittee on Taxonomy. Pain. 1979;6:249.

15. Buck R, Miller RE, Caul WF. Sex, personality, and physiological variables in the communication of affect via facial expression. J Pers Soc Psychol. 1974;30:587-96. 
16. Burokas L. Factors affecting nurses' decisions to medicate pediatric patients after surgery. Heart Lung. 1985;14:373-9.

17. Lander J, Fowler-Kerry S, Hargreaves A. Gender effects in pain perception. Percept Mot Skills. 1989;68(3 Pt 2):1088-90.

18. Hamers JP, van den Hout MA, Halfens RJ, Abu-Saad HH, Heijltjes AE. Differences in pain assessment and decisions regarding the administration of analgesics between novices, intermediates and experts in pediatric nursing. Int J Nurs Stud. 1997;34:325-34.

19. Porter FL, Wolf CM, Gold J, Lotsoff D, Miller JP. Pain and pain management in newborn infants: a survey of physicians and nurses. Pediatrics. 1997;100:626-32.

20. Craig KD. The facial display of pain. In: Finley GA, McGrath PJ, editors. Measurement of pain in infants and children. Seattle: IASP; 1998. p. 103-22.
21. Prestes AC, Guinsburg R, Balda RC, Marba ST, Rugolo LM, Pachi PR, et al. Freqüência de emprego de analgésicos em unidades de terapia intensive neonatal universitárias. J Pediatr (Rio J). 2005;81:405-10.

22. Karraker $\mathrm{KH}$. Adult attention to infants in a newborn nursery. Nurs Res. 1986;35:358-63.

23. Hadjistavropoulos HD, Ross MA, Von Baeyer CL. Are physicians' ratings of pain affected by patients' physical attractiveness? Soc Sci Med. 1990;31:69-72.

Correspondence:

Ruth Guinsburg

Rua Vicente Felix, 77/09

CEP 01410-020 - São Paulo, SP - Brazil

Tel.: +55 (11) 5579.4982, +55 (11) 5579.1676

E-mail: ruthgbr@netpoint.com.br 